\title{
C-Reactive Protein-based Prognostic Measures Are Superior at Predicting Survival Compared with Peripheral Blood Cell Count-based Ones in Patients After Curative Resection for Pancreatic Cancer
}

\author{
YUKI FUJIWARA, KOICHIRO HARUKI, HIROAKI SHIBA, RYOGA HAMURA, TAKASHI HORIUCHI, \\ YOSHIHIRO SHIRAI, KENEI FURUKAWA, TAKESHI GOCHO and KATSUHIKO YANAGA \\ Department of Surgery, The Jikei University School of Medicine, Tokyo, Japan
}

\begin{abstract}
Aim: Prognostic factors of recurrence and survival in various cancer types have been reported and include $C$ reactive protein (CRP)-based measures as evidenced by the Glasgow prognostic score (GPS), as well as peripheral blood cell-based prognostic values such as the prognostic index (PI), neutrophil-to-lymphocyte ratio (NLR), and platelet-tolymphocyte ratio (PLR). The aim of this study was to identify significant prognostic values and compare them for suitability for use in patients after curative pancreatic resection for pancreatic cancer. Materials and Methods: Between 2000 and 2015, 188 patients were enrolled in this retrospective study. The relationship between clinicopathological variables including various prognostic values and disease-free (DFS) and overall $(O S)$ survival was investigated by univariate analysis. The area under the receiver operating characteristics curve $(A U C)$ was evaluated to compare the predictive ability of each of these scoring systems. Multivariate analysis was then performed to identify clinicopathological variables that associated DFS and OS. Results: In univariate analysis, GPS, modified GPS, CRP to albumin ratio and PI were significant risk factors for both DFS and OS. The AUC of CRP-based scores (GPS, modified GPS, and CRP to albumin ratio) were consistently larger in comparison with PI, which consists of both CRP and peripheral blood cell scores, at all time points for both DFS and OS. In multivariate analysis, GPS was the only independent risk factor of tumor recurrence and survival. Conclusion: CRP-based prognostic scores have an independent
\end{abstract}

Correspondence to: Yuki Fujiwara, MD, The Jikei University School of Medicine, 3-25-8, Nishi-Shinbashi, Minato-ku, Tokyo 105-8461, Japan. Tel: +81 334331111 ext. 2345, Fax: +81 334331230, e-mail: sheetan@jikei.ac.jp

Key Words: Pancreatic cancer, C-reactive protein, CRP-based prognostic values, Glasgow prognostic score. value for both tumor recurrence and prognosis in patients after curative resection for pancreatic cancer, and are superior to other peripheral blood cell count-based prognostic scores.

Pancreatic carcinoma is one of the most fatal human malignant digestive cancers and the fourth leading cause of cancer-related death worldwide (1). Although elective surgical pancreatic resection is the curative treatment for pancreatic cancer, only $10-15 \%$ of patients with pancreatic cancer are able to undergo pancreatic resection (2). However, the overall survival rate of patients who undergo such curative surgical resection remains poor in spite of improvements in surgical techniques, instruments, and postoperative management $(3,4)$. Prognostic factors for long-term survival in patients undergoing resection of pancreatic cancer include small tumor size, absence of lymph node involvement, curative (R0) resection and the absence of adjuvant chemotherapy (5-7).

Recently, the prognostic outcomes of patients with cancer have been reported to be associated not only with tumorrelated factors but also with host-related factors. Systemic inflammation-based values have been determined in patients with different resectable malignancies as independent factors of tumor recurrence and tumor-related prognosis. These values can generally be divided into three groups: I: The Glasgow prognostic score (GPS) (8-10), modified GPS (mGPS), and C-reactive protein (CRP) to albumin ratio (CRP/Alb ratio) $(11,12)$ are based on CRP; II: The neutrophil to lymphocyte ratio (NLR) (13) and platelet to lymphocyte ratio (PLR) (14) are based on counts of peripheral blood cell components; III: The prognostic index (PI) derived by combination of serum albumin and peripheral lymphocyte count (15), and the prognostic nutritional index score (PNI) derived by combination of serum CRP and white blood cell (WBC) count (16) are based on peripheral blood cell components. 
The aim of this retrospective study was to determine the most clinically useful prognostic score by comparing these inflammation-based prognostic scores in patients after curative pancreatic resection for pancreatic cancer.

\section{Materials and Methods}

Patients. Between 2000 and 2015, 195 patients with primary pancreatic cancer underwent curative pancreatic resection with lymphadenectomy at the Department of Surgery, The Jikei University Hospital, Tokyo, Japan. Out of these, seven patients were subsequently excluded due to loss to follow-up in the early postoperative period, leaving the remaining 188 patients for this retrospective study.

Recurrence of tumor was defined as newly detected local or distant metastatic tumors by imaging modalities consisting of ultrasonography, computed tomography, or magnetic resonance imaging with or without increase in serum carcinoembryonic antigen (CEA) or serum carbohydrate antigen 19-9 (CA19-9).

Blood samples were obtained before elective pancreatic resection and hemograms, including absolute WBC, neutrophil, lymphocyte, monocyte and platelet counts, as well as serum albumin and CRP were routinely measured for each patient.

Definition of prognostic values. In order to compare the prognostic variables, definitions of GPS, mGPS, NLR, PLR, CRP/Alb ratio, PI and PNI are listed in Table I.

Relationship between prognostic values and clinicopathological variables. The relationship between clinicopathological findings and disease-free (DFS) and overall (OS) survival was investigated in patients with primary pancreatic cancer after curative pancreatic resection by univariate analysis. The following 28 variables were evaluated: Age, gender, type of operation, reconstruction of portal vein, duration of operation, intraoperative blood loss, body mass index (BMI), postoperative pancreatic fistula, postoperative complication based on Clavien-Dindo classification (17), intraoperative red blood cell concentrate or fresh frozen plasma transfusion, postoperative hospital stay, diabetes mellitus, serum CEA, serum CA19-9, neutrophil count, lymphocyte count, monocyte count, tumor differentiation, type of tumor, TNM classification based on the Union for International Cancer Control (UICC) 8th edition (18), resected margin, GPS, mGPS, NLR, PLR, CRP/Alb ratio, PNI and PI.

Receiver operating characteristics (ROC) curves were constructed for DFS and OS to compare those which were significant factors in univariate analysis. Four timepoints were used: 6, 12, 18 and 24 months for DFS status; and 12, 24, 36 and 60 for OS status. The areas under the ROC curve (AUC) of these scores were statistically compared at all these time points for both DFS and OS to determine which value was superior in both CRP-based and peripheral blood cell-based prognostic values.

Next, we compared the most useful prognostic value with clinical variables, and determined the independent prognostic factors in both DFS and OS by multivariate analysis. The evaluated factors consisted of the following: reconstruction of the portal vein, preoperative serum CA19-9, tumor differentiation, type of tumor, TNM classification, resected margin and GPS for DFS; reconstruction of the portal vein, preoperative serum CEA, preoperative serum CA19-9, tumor differentiation, type of tumor, resected margin and GPS for OS.
Table I. Prognostic scores based on serum or blood cellular components.

\begin{tabular}{|c|c|c|}
\hline Index & & Score \\
\hline \multirow[t]{4}{*}{ GPS } & $\mathrm{CRP}(\leq 1.0 \mathrm{mg} / \mathrm{dl})+\mathrm{Alb}(\geq 3.5 \mathrm{~g} / \mathrm{dl})$ & 0 \\
\hline & $\mathrm{CRP}(\leq 1.0 \mathrm{mg} / \mathrm{dl})+\mathrm{Alb}(<3.5 \mathrm{~g} / \mathrm{dl})$ & 1 \\
\hline & $\mathrm{CRP}(>1.0 \mathrm{mg} / \mathrm{dl})+\mathrm{Alb}(\geq 3.5 \mathrm{~g} / \mathrm{dl})$ & 1 \\
\hline & $\mathrm{CRP}(>1.0 \mathrm{mg} / \mathrm{dl})+\mathrm{Alb}(<3.5 \mathrm{~g} / \mathrm{dl})$ & 2 \\
\hline \multirow[t]{4}{*}{ mGPS } & $\mathrm{CRP}(\leq 1.0 \mathrm{mg} / \mathrm{dl})+\mathrm{Alb}(\geq 3.5 \mathrm{~g} / \mathrm{dl})$ & 0 \\
\hline & $\mathrm{CRP}(\leq 1.0 \mathrm{mg} / \mathrm{dl})+\mathrm{Alb}(<3.5 \mathrm{~g} / \mathrm{dl})$ & 0 \\
\hline & $\mathrm{CRP}(>1.0 \mathrm{mg} / \mathrm{dl})+\mathrm{Alb}(\geq 3.5 \mathrm{~g} / \mathrm{dl})$ & 1 \\
\hline & $\mathrm{CRP}(>1.0 \mathrm{mg} / \mathrm{dl})+\mathrm{Alb}(<3.5 \mathrm{~g} / \mathrm{dl})$ & 2 \\
\hline \multirow[t]{2}{*}{ NLR } & Neutrophil:lymphocyte count $<5: 1$ & 0 \\
\hline & Neutrophil:lymphocyte count $\geq 5: 1$ & 1 \\
\hline \multirow[t]{3}{*}{ PLR } & Platelet/lymphocyte count $<150$ & 0 \\
\hline & Platelet count /lymphocyte count $150-<300$ & 1 \\
\hline & Platelet count/lymphocyte count $\geq 300$ & 2 \\
\hline \multirow[t]{2}{*}{$\mathrm{CRP} / \mathrm{Alb}$ ratio } & $\mathrm{CRP} / \mathrm{Alb} \leq 0.004$ & 0 \\
\hline & $\mathrm{CRP} / \mathrm{Alb}>0.004$ & 1 \\
\hline \multirow[t]{4}{*}{ PI } & $\mathrm{CRP}(\leq 1.0 \mathrm{mg} / \mathrm{dl})+\mathrm{WBC}$ count $\left(\leq 7.0 \times 10^{3} / \mu \mathrm{l}\right)$ & 0 \\
\hline & $\mathrm{CRP}(>1.0 \mathrm{mg} / \mathrm{dl})+\mathrm{WBC}$ count $\left(\leq 7.0 \times 10^{3} / \mu \mathrm{l}\right)$ & 1 \\
\hline & $\mathrm{CRP}(\leq 1.0 \mathrm{mg} / \mathrm{dl})+\mathrm{WBC}$ count $\left(>7.0 \times 10^{3} / \mu \mathrm{l}\right)$ & 1 \\
\hline & $\mathrm{CRP}(>1.0 \mathrm{mg} / \mathrm{dl})+\mathrm{WBC}$ count $\left(>7.0 \times 10^{3} / \mu \mathrm{l}\right)$ & 2 \\
\hline \multirow[t]{2}{*}{ PNI } & {$[$ Alb $(\mathrm{g} / \mathrm{dl}) \times 10]+\left[5 \times\right.$ Iymphocyte count $\left.\left(/ 10^{3}\right)\right] \geq 45$} & 0 \\
\hline & {$[\mathrm{Alb}(\mathrm{g} / \mathrm{dl}) \times 10]+\left[5 \times\right.$ lymphocyte count $\left.\left(/ 10^{3}\right)\right]<45$} & 1 \\
\hline
\end{tabular}

Alb: Serum albumin; CRP: serum C-reactive protein; GPS: Glasgow prognostic score; mGPS: modified Glasgow prognostic score; NLR: neutrophil lymphocyte ratio score; PI: Prognostic index; PLR: platelet to lymphocyte ratio score; PNI: prognostic nutritional index; WBC: white blood cell.

Pancreatic fistula was defined by the guideline of the International Study Group on Pancreatic Fistula (ISGPF) (19). Pancreatic fistula was classified into three categories by ISGPF as follows: Transient pancreatic fistula (no clinical impact) (grade A); fistula requiring a change in management or adjustment in the clinical pathway (grade B); and fistula requiring a major change in clinical management or deviation from the normal clinical pathway (grade C). In the current study, grade $\mathrm{B}$ and $\mathrm{C}$ were defined as postoperative pancreatic fistula (POPF). Postoperative complications were defined by Clavien-Dindo classification and divided into two groups: grade II or less; and grade IIIa or more. Use of blood products and dose were determined by the preference of attending surgeons based on guidelines for administration of blood products by the Japanese Ministry of Health and Welfare (20), as well as intraoperative blood loss, postoperative hemoglobin, serum albumin, and prothrombin time.

This retrospective study was approved by the Ethics Committee of the Jikei University School of Medicine (no. 21-121).

Statistical analysis. Data are expressed as a mean \pm standard deviation (SD), or frequency. Univariate analysis of patient characteristics was performed using Mann-Whitney's $U$-test or chisquare test. Both univariate and multivariate analysis of DFS and OS were performed using Cox proportional regression models with backward elimination stepwise approach. The software package SPSS (version 20; IBM SPSS statistics ${ }^{\circledR}$, Tokyo, Japan) was used for statistical analyses. All $p$-values were considered statistically significant when the probability of association was less than 0.05 . 
Table II. Patient characteristics.

\begin{tabular}{|c|c|c|}
\hline \multicolumn{2}{|l|}{ Characteristic } & \multirow[b]{2}{*}{$67.0 \pm 9.87(27-84)$} \\
\hline Age, years & Mean \pm SD (range) & \\
\hline Gender, $\mathrm{n}$ & Male: female & $115: 73$ \\
\hline Disease-free survival, years & Median $(95 \% \mathrm{CI})$ & $1.07(0.90-1.38)$ \\
\hline Overall survival, years & Median $(95 \% \mathrm{CI})$ & $2.14(1.92-2.90)$ \\
\hline Duration of operation, min & Mean \pm SD (range) & $497.6 \pm 144.6(140-992)$ \\
\hline Intraoperative blood loss, $\mathrm{ml}$ & Mean $\pm \mathrm{SD}$ (range) & $1,183.2 \pm 1,457.6(0-12,320)$ \\
\hline Postoperative hospital stay, days & Mean $\pm \mathrm{SD}$ (range) & $30.6 \pm 42.6(8-419)$ \\
\hline Preoperative serum CEA, ng/ml & Mean \pm SD (range) & $7.86 \pm 22.6(4-286)$ \\
\hline Preoperative serum CA19-9, U/ml & Mean \pm SD (range) & $277.2 \pm 601.9(1-5,750)$ \\
\hline Preoperative neutrophil count, $\times 10^{3} / \mu 1$ & Mean \pm SD (range) & $3.65 \pm 2.0(0.9-18.3)$ \\
\hline Preoperative lymphocyte count, $\times 10^{3} / \mu 1$ & Mean \pm SD (range) & $1.51 \pm 0.53(0.6-4.0)$ \\
\hline Preoperative serum Alb, g/dl & Mean \pm SD (range) & $3.84 \pm 0.49(2.7-4.9)$ \\
\hline Preoperative serum CRP, mg/dl & Mean \pm SD (range) & $0.789 \pm 1.8(0.04-14.7)$ \\
\hline TNM classification, $\mathrm{n}^{*}$ & 0: I: II: III: IV & 4:53:96:31:4 \\
\hline
\end{tabular}

Alb: Albumin; CA19-9, carbohydrate antigen 19-9; CEA, carcinoembryonic antigen; CI: confidence interval; CRP, C-reactive protein. *Based on 8th edition Union for International Cancer Control (18).

\section{Results}

Patient characteristics. Table II shows the patient characteristics. The mean age was 67.0 years (range $=27-84$ years). The operative procedures consisted of pancreaticoduodenectomy in 125 , distal pancreatectomy in 57 , central pancreatectomy in two and total pancreatectomy in four patients. The median of DFS and OS were 1.14 and 2.41 years, respectively. The 1-, 3-and 5-year DFS and OS rates were $56.1 \%, 27.0 \%$ and $18.3 \%$, and $80.2 \%, 43.5 \%$ and $29.0 \%$, respectively.

Univariate analysis of clinicopathological variables in relation to survival in patients after curative resection for pancreatic cancer. Table III lists the univariate analysis of the relationship between clinicopathological variables and DFS as well as OS after curative pancreatic resection. In univariate analysis of DFS, reconstruction of portal vein $(p=0.002) ;$ preoperative serum CA19-9 $\geq 200 \mathrm{U} / \mathrm{ml}$ $(p<0.001)$; not well-differentiated tumor $(p<0.001)$; infiltrative tumor type $(p=0.002)$; advanced TNM stage $(p<0.001)$; positive resected margin $(p=0.011)$; GPS score 1 or 2 (Figure $1 \mathrm{~A} ; p=0.008$ and $p<0.001$, respectively); mGPS score 1 or 2 (Figure 1B; both $p<0.001$ ); CRP/Alb ratio score 1 (Figure $1 C ; p=0.003$ ); and PI score 2 (Figure $1 \mathrm{D} ; p=0.006)$ were significant factors for cancer recurrence. For OS, reconstruction of portal vein $(p=0.017)$; preoperative serum CEA $\geq 10 \mathrm{ng} / \mathrm{ml}$ ( $p=0.044)$; preoperative serum CA19-9 $\geq 200 \mathrm{U} / \mathrm{ml}$ ( $p=0.003)$; not well-differentiated tumor $(p=0.001)$; infiltrative tumor type $(p=0.025)$; advanced TNM stage $(p=0.002)$; positive resected margin $(p=0.027)$; GPS score 1 or 2 (Figure 1E; $p=0.002$ and $p<0.001$ respectively); mGPS score 1 or 2 (Figure $1 \mathrm{~F}$; $p<0.001$ ); CRP/Alb ratio score 1 (Figure 1G; $p<0.001$ ); PI score 1 or 2 (Figure $1 \mathrm{H} ; p=0.002$ and $p<0.006$, respectively) were significantly associated with poor outcome.

Comparison of AUC for prognostic value. Figure 2 shows the ROC curves for DFS at 6,12, 18 and 24 months and for OS at 12, 24, 36 and 60 months using scores for GPS, mGPS, $\mathrm{CRP} / \mathrm{Alb}$ ratio score and PI score, which were significantly associated with both DFS and OS in the univariate analysis. The comparison of AUC to assess the discriminatory ability of each scoring system is shown in Table IV. The AUC for scores for CRP-based measures GPS, mGPS and CRP/Alb ratio were consistently larger in comparison with the peripheral blood cell count-based PI score at all time points for both DFS and OS. This showed that CRP-based prognostic measures such as GPS score, mGPS score and $\mathrm{CRP} / \mathrm{Alb}$ ratio were superior to other prognostic scores for PI, PNI, NLR and PLR at predicting tumor recurrence as well as prognosis in patients with pancreatic cancer.

Multivariate analysis of clinicopathological variables in relation to survival in patients after curative resection for pancreatic cancer. Table $\mathrm{V}$ lists multivariate analysis of the relationship of the clinicopathological variables with GPS score and DFS as well as OS after curative pancreatic resection. In multivariate analysis of DFS, preoperative serum CA19-9 $\geq 200$ $\mathrm{U} / \mathrm{ml}(p=0.016)$, not well-differentiated tumor $(p=0.001)$, advanced TNM stage $(p=0.027)$, GPS score $1(p=0.015)$ and GPS score $2(p=0.001)$ were independent risk factors of cancer recurrence. In OS, preoperative serum CEA $\geq 10 \mathrm{ng} / \mathrm{ml}$ of $(p=0.008)$, not well-differentiated tumor $(p=0.006)$, advanced 
Table III. Univariate analysis of disease-free and overall survival in patients with pancreatic cancer after pancreatic resection.

\begin{tabular}{|c|c|c|c|c|c|c|}
\hline \multirow[b]{2}{*}{ Factor } & \multirow[b]{2}{*}{ Subgroup } & \multirow[t]{2}{*}{$\mathrm{N}$} & \multicolumn{2}{|c|}{ Disease-free survival } & \multicolumn{2}{|c|}{ Overall survival } \\
\hline & & & Hazard ratio $(95 \% \mathrm{CI})$ & $p$-Value & Hazard ratio $(95 \% \mathrm{CI})$ & $p$-Value \\
\hline \multirow[t]{2}{*}{ Age } & $<70$ Years & 102 & Ref & & Ref & \\
\hline & $\geq 70$ Years & 86 & $0.744(0.535-1.036)$ & 0.080 & $0.831(0.581-1.188)$ & 0.309 \\
\hline \multirow[t]{2}{*}{ Gender } & Male & 115 & Ref & & Ref & \\
\hline & Female & 73 & $0.909(0.650-1.272)$ & 0.909 & $0.846(0.588-1.217)$ & 0.367 \\
\hline \multirow[t]{2}{*}{ Type of operation } & $\mathrm{PD}$ & 125 & Ref & & Ref & \\
\hline & Other & 63 & $0.767(0.539-1.090)$ & 0.139 & $0.735(0.503-1.073)$ & 0.111 \\
\hline \multirow[t]{2}{*}{ Portal vein reconstruction } & No & 155 & Ref & & Ref & \\
\hline & Yes & 33 & $1.899(1.274-2.830)$ & 0.002 & $1.698(1.098-2624)$ & 0.017 \\
\hline \multirow[t]{2}{*}{ Duration of operation } & $<500 \min$ & 94 & Ref & & Ref & \\
\hline & $\geq 500 \mathrm{~min}$ & 94 & $1.064(0.767-1.475)$ & 0.710 & $1.036(0.729-1.472)$ & 0.844 \\
\hline \multirow{2}{*}{ Intraoperative blood loss } & $<800 \mathrm{ml}$ & 96 & Ref & & Ref & \\
\hline & $\geq 800 \mathrm{ml}$ & 92 & $1.013(0.731-1.403)$ & 0.938 & $1.220(0.859-1.735)$ & 0.267 \\
\hline \multirow[t]{2}{*}{ BMI } & $<25 \mathrm{~kg} / \mathrm{m}^{2}$ & 158 & Ref & & Ref & \\
\hline & $\geq 25 \mathrm{~kg} / \mathrm{m}^{2}$ & 30 & $0.720(0.453-1.144)$ & 0.165 & $0.740(0.454-1.208)$ & 0.229 \\
\hline \multirow[t]{2}{*}{ POPF (grade B or C) } & No & 159 & Ref & & Ref & \\
\hline & Yes & 29 & $1.001(0.635-1.576)$ & 0.998 & $1.188(0.743-1.900)$ & 0.471 \\
\hline \multirow[t]{2}{*}{ Postoperative complications* } & $\leq$ Grade II & 146 & Ref & & Ref & \\
\hline & $\geq$ Grade IIIa & 42 & $1.298(0.879-1.915)$ & 0.190 & $1.210(0.789-1.856)$ & 0.383 \\
\hline \multirow[t]{2}{*}{ Intraoperative RCC or FFP transfusion } & No & 133 & Ref & & Ref & \\
\hline & Yes & 55 & $1.153(0.809-1.644)$ & 0.430 & $1.375(0.946-1.999)$ & 0.095 \\
\hline \multirow[t]{2}{*}{ Postoperative hospital stay } & $<30$ Days & 131 & Ref & & Ref & \\
\hline & $\geq 30$ Days & 57 & $1.233(0.867-1.754)$ & 0.243 & $1.149(0.787-1.678)$ & 0.473 \\
\hline \multirow[t]{2}{*}{ Diabete mellitus } & No & 119 & Ref & & Ref & \\
\hline & Yes & 69 & $0.915(0.650-1.287)$ & 0.609 & $0.901(0.622-1.304)$ & 0.581 \\
\hline Preoperative serum CEA & $<10 \mathrm{ng} / \mathrm{ml}$ & 163 & Ref & & Ref & \\
\hline & $\geq 10 \mathrm{ng} / \mathrm{ml}$ & 25 & $1.440(0.888-2.335)$ & 0.139 & $1.654(1.013-2.699)$ & 0.044 \\
\hline Preoperative serum CA19-9 & $<200 \mathrm{U} / \mathrm{ml}$ & 131 & Ref & & Ref & \\
\hline & $\geq 200 \mathrm{U} / \mathrm{ml}$ & 57 & $1.993(1.408-2.823)$ & $<0.001$ & $1.762(1.217-2.551)$ & 0.003 \\
\hline Preoperative neutrophil count & $<3.0 \times 10^{3} / \mu 1$ & 80 & Ref & & Ref & \\
\hline & $\geq 3.0 \times 10^{3} / \mu 1$ & 108 & $0.790(0.569-1.097)$ & 0.159 & $0.809(0.568-1.151)$ & 0.239 \\
\hline Preoperative lymphocyte count & $<2.0 \times 10^{3} / \mu \mathrm{l}$ & 29 & Ref & & Ref & \\
\hline & $\geq 2.0 \times 10^{3} / \mu 1$ & 159 & $1.430(0.891-2.294)$ & 0.139 & $1.470(0.890-2.427)$ & 0.132 \\
\hline Preoperative monocyte count & $<300 / \mu 1$ & 80 & Ref & & Ref & \\
\hline & $\geq 300 / \mu 1$ & 108 & $0.953(0.686-1.323)$ & 0.772 & $0.854(0.601-1.213)$ & 0.378 \\
\hline Tumor differentiation & Well-differentiated & 73 & Ref & & Ref & \\
\hline & Other & 115 & $2.110(1.481-3.005)$ & $<0.001$ & $1.870(1.284-2.724)$ & 0.001 \\
\hline Type of tumor & Other & 64 & Ref & & Ref & \\
\hline & Infiltrative type & 124 & $1.751(1.221-2.511)$ & 0.002 & $1.550(1.058-2.272)$ & 0.025 \\
\hline TNM Stage** & 0, I or II & 153 & Ref & & Ref & \\
\hline & III or IV & 35 & $1.885(1.345-2.641)$ & $<0.001$ & $1.976(1.290-3.027)$ & 0.002 \\
\hline Resected margin & R0 & 133 & Ref & & Ref & \\
\hline & $\mathrm{R} 1$ or $\mathrm{R} 2$ & 55 & $1.574(1.110-2.232)$ & 0.011 & $1.535(1.051-2.243)$ & 0.027 \\
\hline GPS & 0 & 123 & Ref & & Ref & \\
\hline & 1 & 38 & $1.717(1.151-2.562)$ & 0.008 & $1.976(1.282-3.047)$ & 0.002 \\
\hline & 2 & 27 & $3.071(1.925-4.900)$ & $<0.001$ & $4.082(2.564-6.498)$ & $<0.001$ \\
\hline mGPS & 0 & 140 & Ref & & Ref & \\
\hline & 1 & 21 & $2.498(1.541-4.049)$ & $<0.001$ & $2.463(1.436-4.224)$ & 0.001 \\
\hline & 2 & 27 & $3.043(1.918-4.826)$ & $<0.001$ & $3.880(2.460-6.121)$ & $<0.001$ \\
\hline NLR & 0 & 171 & Ref & & Ref & \\
\hline & 1 & 17 & $0.799(0.431-1.479)$ & 0.475 & $0.993(0.534-1.845)$ & 0.982 \\
\hline PLR & 0 & 90 & Ref & & Ref & \\
\hline & 1 & 87 & $1.136(0.521-2.475)$ & 0.749 & $0.815(0.371-1.791)$ & 0.611 \\
\hline & 2 & 11 & $1.510(0.693-3.289)$ & 0.299 & $1.184(0.541-2.587)$ & 0.673 \\
\hline $\mathrm{CRP} / \mathrm{Alb}$ ratio score & 0 & 100 & Ref & & Ref & \\
\hline & 1 & 88 & $1.655(1.193-2.294)$ & 0.003 & $1.928(1.355-2.744)$ & $<0.001$ \\
\hline
\end{tabular}




\begin{tabular}{|c|c|c|c|c|c|c|}
\hline \multirow[b]{2}{*}{ Factor } & \multirow[b]{2}{*}{ Subgroup } & \multirow[t]{2}{*}{$\mathrm{N}$} & \multicolumn{2}{|c|}{ Disease-free survival } & \multicolumn{2}{|c|}{ Overall survival } \\
\hline & & & Hazard ratio $(95 \% \mathrm{CI})$ & $p$-Value & Hazard ratio $(95 \% \mathrm{CI})$ & $p$-Value \\
\hline \multirow[t]{2}{*}{ PNI } & 0 & 63 & Ref & & Ref & \\
\hline & 1 & 125 & $1.165(0.818-1.658)$ & 0.397 & $0.983(0.675-1.429)$ & 0.927 \\
\hline \multirow[t]{3}{*}{ PI } & 0 & 132 & Ref & & Ref & \\
\hline & 1 & 39 & $1.441(0.953-2.180)$ & 0.083 & $1.981(1.293-3.033)$ & 0.002 \\
\hline & 2 & 17 & $2.190(1.248-3.841)$ & 0.006 & $2.798(1.581-4.953)$ & $<0.001$ \\
\hline
\end{tabular}

Alb: Serum albumin; BMI: body mass index; CA19-9: carbohydrate antigen 19-9; CEA: carcinoembryonic antigen; CRP: serum C-reactive protein; FFP: fresh frozen plasma; GPS: Glasgow prognostic score; mGPS: modified Glasgow prognostic score; NLR: neutrophil lymphocyte ratio score; PI: Prognostic index; PLR: platelet to lymphocyte ratio score; PNI: prognostic nutritional index; PD: pancreaticoduodenectomy; POPF: postoperative pancreatic fistula; RCC: red blood cell concentrate. ${ }^{*}$ Clavien-Dindo classification (17). **Based on 8th edition Union for International Cancer Control (18).

A

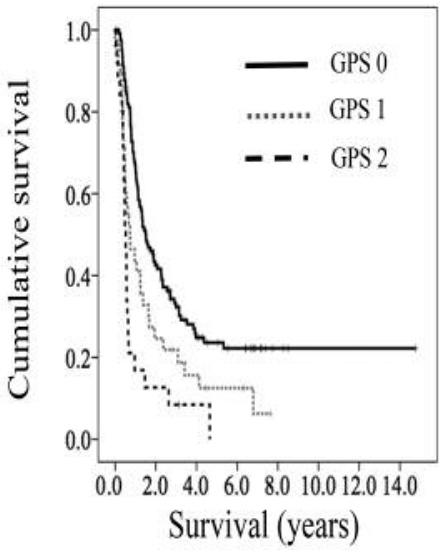

B

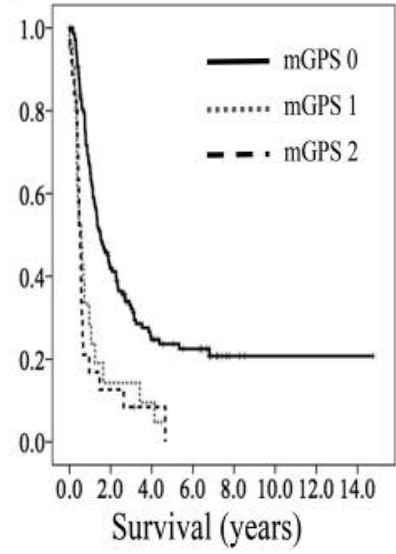

C

G

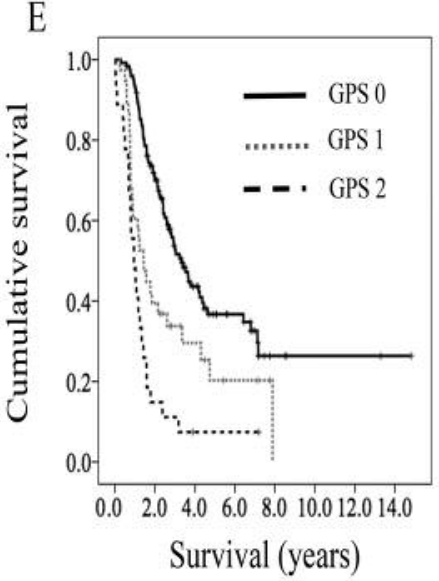

F

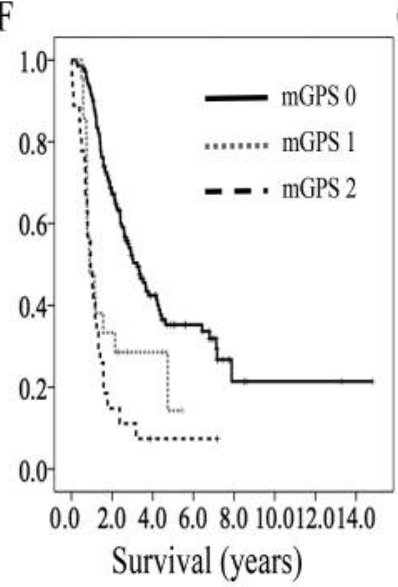

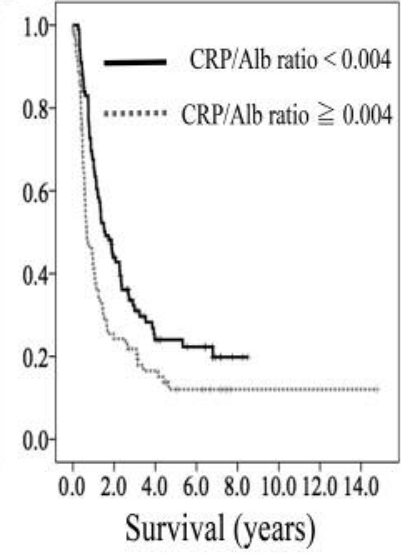

D

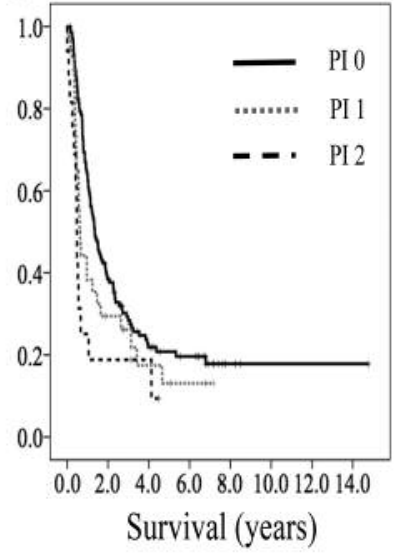

H

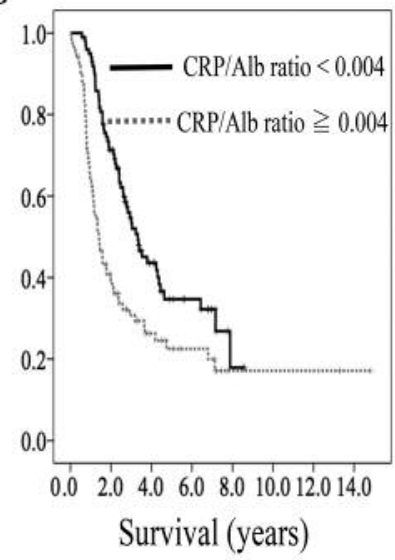

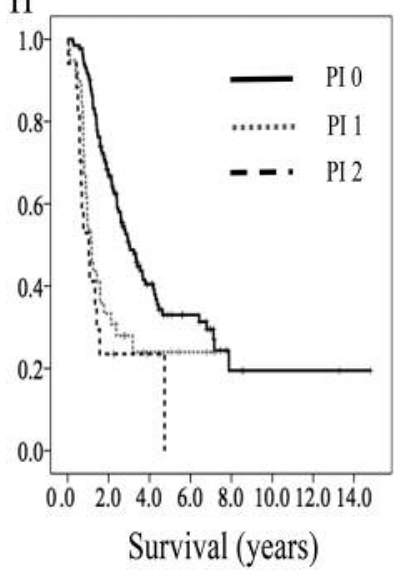

Figure 1. Disease-free (A-D) and overall survival (E-H) in patients with pancreatic cancer according to prognostic measures: Glasgow prognostic score $(G P S)(A, E)$, modified GPS $(B, F)$, serum $C$-reactive protein $(C R P) /$ serum albumin $(A l b)$ ratio $(C, G)$ and prognostic index $(P I)(D, H)$. 

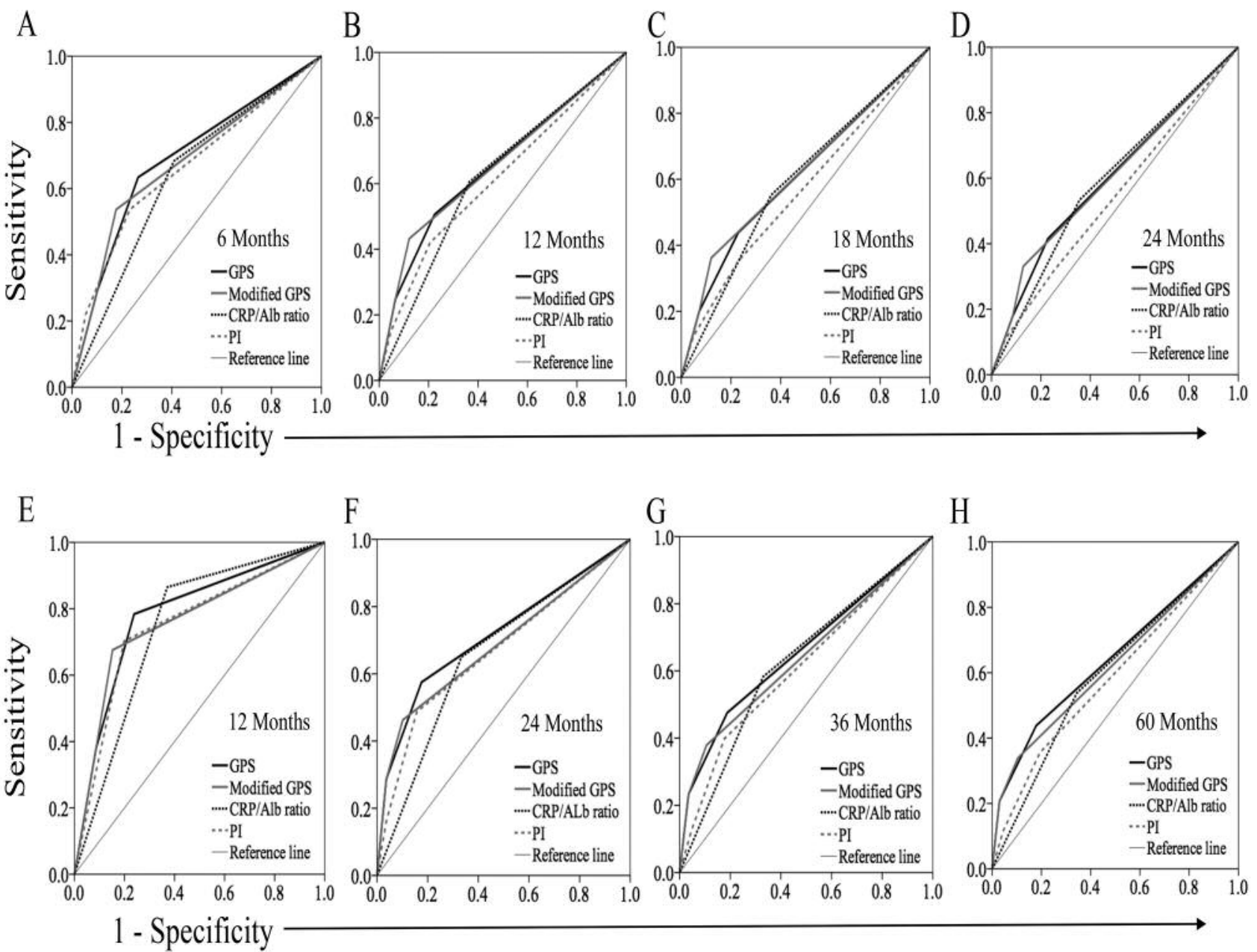

G

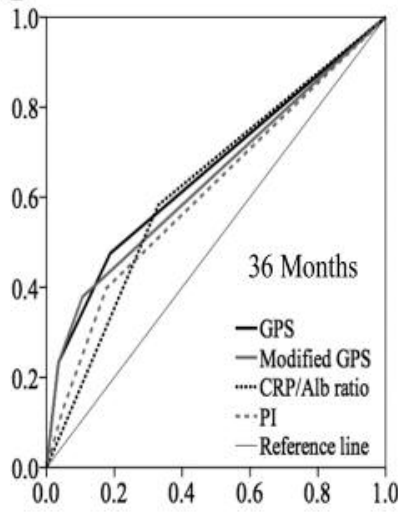

$\mathrm{H}$

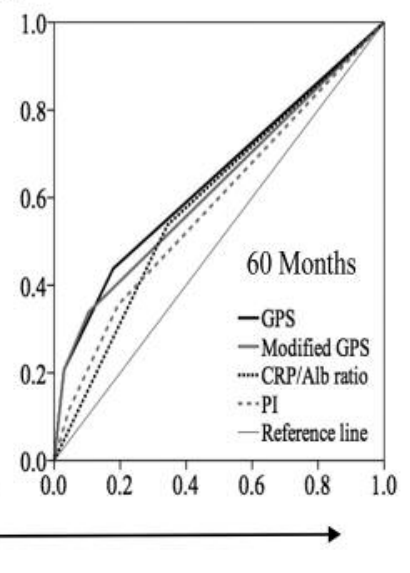

Figure 2. Receiver operating characteristics curve according to prognostic measures for disease-free outcome prediction at 6 (A), 12 (B), 18 (C) and $24(D)$ months and for overall mortality at $12(E), 24(F), 36(G)$ and $60(H)$ months.

TNM stage $(p=0.024)$, and GPS $(p=0.002)$ or $2(p<0.001)$ were independent risk factors of poor prognosis.

\section{Discussion}

The antitumor host immune response has an important role in progression and prognosis of a tumor. The intimate interaction of inflammation and tumor biology has been wellrecognized (21-23). Cancer-related inflammation promotes proliferation of tumor cells, tumor angiogenesis, and invasion and metastasis of cancer cells because of activation by interleukin-1, interleukin-6 as well as tumor necrosis factor $\alpha$, and an increase in the number of regulatory T-lymphocytes $(24,25)$. In fact, some types of pro-inflammatory cytokines such as interleukin-1 and interleukin-6 are increased in patients with pancreatic cancer (26).
GPS was first reported as an independent prognostic factor for patients with inoperable lung cancer in 2003 (27). Some studies reported that GPS predicted tumor-related prognosis not only in patients with various advanced and unresectable gastrointestinal cancer with poor performance status and severe body weight loss (28-30), but also in those with various types of resectable digestive cancer (8-10). The peripheral blood cell component-based values as represented by NLR and PLR have already been reported as prognostic factors of tumor-related outcome $(13,14,31)$. A high neutrophil count in peripheral blood contributes to tumor angiogenesis due to their acting as important compartments for circulating vascular endothelial growth factor (32). Platelets are often activated by tumor cells and release several types of cytokines that promote angiogenesis (33). Moreover, immune response against tumors depends on the number of 
Table IV. Comparison of area under the curve (AUC) for survival of patients after pancreatic resection using serum C-reactive protein-based prognostic scores.

\begin{tabular}{|c|c|c|c|c|c|c|c|}
\hline \multirow[b]{2}{*}{ Time point } & \multicolumn{3}{|c|}{ Disease free survival } & \multirow[b]{2}{*}{ Time point } & \multicolumn{3}{|c|}{ Overall survival } \\
\hline & AUC & $95 \% \mathrm{CI}$ & $p$-Value & & AUC & $95 \% \mathrm{CI}$ & $p$-Value \\
\hline \multicolumn{8}{|l|}{6 Months } \\
\hline GPS & 0.691 & $0.595-0.787$ & $<0.001$ & \multirow[t]{4}{*}{12 Months } & 0.784 & $0.697-0.871$ & $<0.001$ \\
\hline mGPS & 0.678 & $0.579-0.778$ & $<0.001$ & & 0.761 & $0.666-0.856$ & $<0.001$ \\
\hline $\mathrm{CRP} / \mathrm{Alb}$ ratio & 0.637 & $0.543-0.732$ & 0.007 & & 0.747 & $0.665-0.829$ & $<0.001$ \\
\hline PI & 0.663 & $0.563-0.764$ & 0.001 & & 0.753 & $0.659-0.846$ & $<0.001$ \\
\hline \multicolumn{8}{|l|}{12 Months } \\
\hline GPS & 0.652 & $0.571-0.733$ & $<0.001$ & \multirow[t]{4}{*}{24 Months } & 0.714 & $0.637-0.791$ & $<0.001$ \\
\hline mGPS & 0.656 & $0.575-0.737$ & $<0.001$ & & 0.686 & $0.606-0.766$ & $<0.001$ \\
\hline $\mathrm{CRP} / \mathrm{Alb}$ ratio & 0.620 & $0.539-0.701$ & 0.005 & & 0.658 & $0.579-0.738$ & $<0.001$ \\
\hline PI & 0.612 & $0.530-0.695$ & 0.008 & & 0.669 & $0.588-0.749$ & $<0.001$ \\
\hline \multicolumn{8}{|l|}{18 Months } \\
\hline GPS & 0.612 & $0.532-0.692$ & 0.009 & \multirow[t]{4}{*}{36 Months } & 0.657 & $0.580-0.735$ & $<0.001$ \\
\hline mGPS & 0.620 & $0.540-0.699$ & 0.005 & & 0.642 & $0.564-0.720$ & 0.001 \\
\hline $\mathrm{CRP} / \mathrm{Alb}$ ratio & 0.595 & $0.514-0.677$ & 0.025 & & 0.627 & $0.546-0.707$ & 0.003 \\
\hline PI & 0.567 & $0.486-0.649$ & 0.113 & & 0.613 & $0.532-0.693$ & 0.008 \\
\hline \multicolumn{8}{|l|}{24 Months } \\
\hline GPS & 0.595 & $0.514-0.678$ & 0.028 & \multirow[t]{4}{*}{60 Months } & 0.641 & $0.563-0.720$ & 0.001 \\
\hline mGPS & 0.598 & $0.516-0.680$ & 0.024 & & 0.623 & $0.543-0.702$ & 0.005 \\
\hline $\mathrm{CRP} / \mathrm{Alb}$ ratio & 0.588 & $0.505-0.672$ & 0.043 & & 0.597 & $0.513-0.681$ & 0.028 \\
\hline PI & 0.537 & $0.453-0.622$ & 0.392 & & 0.584 & $0.501-0.667$ & 0.057 \\
\hline
\end{tabular}

Alb: Serum albumin; CI: confidence interval; CRP: serum C-reactive protein; GPS: Glasgow prognostic score; mGPS: modified Glasgow prognostic score; PI: prognostic index.

lymphocytes in peripheral blood (21). In our previous study, PLR ( $\geq 150$ ) was an independent risk factor for both DFS and OS in patients with resectable pancreatic cancer (14). However, in the current study, PLR was not found to be a significant factor of DFS nor OS in univariate analysis. This is because the PLR score was divided into three groups using cut-off values of 150 and 300 to compare with the original PLR scoring system (34). The difference of sample sizes and follow-up duration compared with our previous study may be also one reason to explain this discrepancy.

Analysis and comparison of prognostic variables among the same patient group is very important in order to identify clinically useful scores predicting tumor recurrence and outcome in patients with cancer. In addition, it is also important to analyze prognostic factors in patients with cancer while distinguishing between operable and inoperable cases. A single study showed a comparison of these inflammation-based prognostic values in patients with pancreatic cancer, in which NLR $(>5)$ was superior to the mGPS, PI, PLR and PNI for prognostication (31). Therefore, to the best of our knowledge, the current study is the first report to identify and compare several inflammation-based prognostic values in patients with pancreatic cancer after pancreatic resection.

\section{Conclusion}

CRP-based prognostic scores have an independent value for both tumor recurrence and prognosis in patients after curative pancreatic resection for pancreatic cancer, and are superior to other peripheral blood cell count-based prognostic scores.

\section{Conflicts of Interests}

All Authors declare no conflict of interests in regard to this study.

\section{Ethics approval}

This study was approved by the Ethics Committee of the Jikei University School of Medicine (21-121).

\section{References}

1 Siegel RL, Miller KD and Jemal A: Cancer statistics, 2018. CA Cancer J Clin 68: 7-30, 2018.

2 Bilimoria KY, Bentrem DJ, Ko CY, Stewart AK, Winchester DP and Talamonti MS: National failure to operate on early-stage pancreatic cancer. Ann Surg 246: 173-180, 2007.

3 Cameron JL, Riall TS, Coleman J and Belcher KA: One thousand consecutive pancreaticoduodenectomies. Ann Surg 244: 10-15, 2006. 
Table V. Multivariate analysis of disease-free and overall survival in patients with pancreatic cancer after pancreatic resection.

\begin{tabular}{|c|c|c|c|c|c|}
\hline \multirow[b]{2}{*}{ Factor } & \multirow[t]{2}{*}{$\mathrm{N}$} & \multicolumn{2}{|c|}{ Disease-free survival } & \multicolumn{2}{|c|}{ Overall survival } \\
\hline & & Hazard ratio $(95 \% \mathrm{CI})$ & $p$-Value & Hazard ratio $(95 \% \mathrm{CI})$ & $p$-Value \\
\hline \multicolumn{6}{|c|}{ Portal vein reconstruction } \\
\hline No & 155 & Ref & & Ref & \\
\hline Yes & 33 & $1.260(0.817-1.945)$ & 0.296 & $1.137(0.703-1.839)$ & 0.600 \\
\hline \multicolumn{6}{|c|}{ Preoperative serum CEA } \\
\hline$<10 \mathrm{ng} / \mathrm{ml}$ & 163 & - & & Ref & \\
\hline$\geq 10 \mathrm{ng} / \mathrm{ml}$ & 25 & - & - & $2.001(1.196-3.349)$ & 0.008 \\
\hline \multicolumn{6}{|c|}{ Preoperative serum CA19-9 } \\
\hline$<200 \mathrm{U} / \mathrm{ml}$ & 131 & Ref & & Ref & \\
\hline$\geq 200 \mathrm{U} / \mathrm{ml}$ & 57 & $1.570(1.089-2.261)$ & 0.016 & $1.419(0.961-2.095)$ & 0.078 \\
\hline \multicolumn{6}{|l|}{ Tumor differentiation } \\
\hline WelI-differentiated & 73 & Ref & & Ref & \\
\hline Other & 115 & $1.816(1.259-2.619)$ & 0.001 & $1.736(1.170-2.576)$ & 0.006 \\
\hline \multicolumn{6}{|l|}{ Type of tumor } \\
\hline Other & 64 & Ref & & Ref & \\
\hline Infiltrative type & 124 & $1.279(0.873-1.874)$ & 0.207 & $1.223(0.812-1.842)$ & 0.318 \\
\hline \multicolumn{6}{|l|}{ TNM stage* } \\
\hline 0, I or II & 153 & Ref & & Ref & \\
\hline III or IV & 35 & $1.594(1.053-2.411)$ & 0.027 & $1.684(1.070-2.650)$ & 0.024 \\
\hline \multicolumn{6}{|l|}{ Resected margin } \\
\hline R0 & 133 & Ref & & Ref & \\
\hline $\mathrm{R} 1$ or $\mathrm{R} 2$ & 55 & $1.290(0.895-1.857)$ & 0.172 & $1.224(0.823-1.820)$ & 0.318 \\
\hline \multicolumn{6}{|l|}{ GPS } \\
\hline 0 & 123 & Ref & & Ref & \\
\hline 1 & 38 & $1.667(1.104-2.516)$ & 0.015 & $2.053(1.310-3.217)$ & 0.002 \\
\hline 2 & 27 & $2.396(1.463-3.926)$ & 0.001 & $3.642(2.216-5.987)$ & $<0.001$ \\
\hline
\end{tabular}

CA19-9: Carbohydrate antigen 19-9; CEA: carcinoembryonic antigen; CI: confidence intervaI; GPS: Glasgow prognostic score. *Based on 8th edition Union for International Cancer Control (18).

4 Sohn TA, Yeo CJ, Cameron JL, Koniaris L, Kaushal S, Abrams RA, Sauter PK, Coleman J, Hruban RH and Lillemoe KD: Resected adenocarcinoma of the pancreas-616 patients: Results, outcomes and prognostic indicators. J Gastrointest Surg 4: 567-579, 2000.

5 Han SS, Jang JY, Kim SW, Kim WH, Lee KU and Park YH: Analysis of long-term survivors after surgical resection for pancreatic cancer. Pancreas 32: 271-275, 2006.

6 Howard TJ, Krug JE, Yu J, Zyromski NJ, Schmidt CM, Jacobson LE, Madura JA, Wiebke EA and Lillemoe KD: A marginnegative R0 resection accomplished with minimal postoperative complications is the surgeon's contribution to long-term survival in pancreatic cancer. J Gastrointest Surg 10: 1338-1345; discussion 1345-1336, 2006.

7 Moon HJ, An JY, Heo JS, Choi SH, Joh JW and Kim YI: Predicting survival after surgical resection for pancreatic ductal adenocarcinoma. Pancreas 32: 37-43, 2006.

8 Shiba H, Horiuchi T, Sakamoto T, Furukawa K, Shirai Y, Iida T, Fujiwara Y, Haruki K and Yanaga K: Glasgow prognostic score predicts therapeutic outcome after hepatic resection for hepatocellular carcinoma. Oncol Lett 14: 293-298, 2017.

9 Shiba H, Misawa T, Fujiwara Y, Futagawa Y, Furukawa K, Haruki K, Iwase R, Iida T and Yanaga K: Glasgow prognostic score predicts outcome after surgical resection of gallbladder cancer. World J Surg 39: 753-758, 2015.
10 Shiba H, Misawa T, Fujiwara Y, Futagawa Y, Furukawa K, Haruki K, Iwase R, Wakiyama S, Ishida $\mathrm{Y}$ and Yanaga $\mathrm{K}$ : Glasgow prognostic score predicts therapeutic outcome after pancreaticoduodenectomy for carcinoma of the ampulla of vater. Anticancer Res 33: 2715-2721, 2013.

11 Haruki K, Shiba H, Horiuchi T, Sakamoto T, Gocho T, Fujiwara Y, Furukawa K, Misawa $\mathrm{T}$ and Yanaga K: Impact of the Creactive protein to albumin ratio on long-term outcomes after hepatic resection for colorectal liver metastases. Am J Surg 214: 752-756, 2017.

12 Haruki K, Shiba H, Shirai Y, Horiuchi T, Iwase R, Fujiwara Y, Furukawa K, Misawa T and Yanaga K: The C-reactive protein to albumin ratio predicts long-term outcomes in patients with pancreatic cancer after pancreatic resection. World J Surg 40: 2254-2260, 2016.

13 Haruki K, Shiba H, Horiuchi T, Shirai Y, Iwase R, Fujiwara Y, Furukawa K, Misawa T and Yanaga K: Neutrophil to lymphocyte ratio predicts therapeutic outcome after pancreaticoduodenectomy for carcinoma of the ampulla of Vater. Anticancer Res 36: 403408, 2016.

14 Shirai Y, Shiba H, Sakamoto T, Horiuchi T, Haruki K, Fujiwara Y, Futagawa Y, Ohashi T and Yanaga K: Preoperative platelet to lymphocyte ratio predicts outcome of patients with pancreatic ductal adenocarcinoma after pancreatic resection. Surgery 158 : 360-365, 2015. 
15 Kasymjanova G, MacDonald N, Agulnik JS, Cohen V, Pepe C, Kreisman H, Sharma R and Small D: The predictive value of pre-treatment inflammatory markers in advanced non-small-cell lung cancer. Curr Oncol 17: 52-58, 2010.

16 Pinato DJ, North BV and Sharma R: A novel, externally validated inflammation-based prognostic algorithm in hepatocellular carcinoma: The prognostic nutritional index (PNI). Br J Cancer 106: 1439-1445, 2012.

17 Clavien PA, Barkun J, de Oliveira ML, Vauthey JN, Dingo D, Schulick RD, de Santibanes E, Pekolj J, Slankamenac K, Bassi C, Graf R, Vonlanthen R, Padbury R, Cameron JL and Makuuchi $\mathrm{M}$ : The Clavien-Dingo classification of surgical complication; five-year experience. Ann Surg 250: 187-196, 2009.

18 Brierley JD, Gospodarowicz MK and Wittekind C: UICC TNM Classification of malignant tumours. 8th ed. Hoboken (New Jersey), US: Wiley-Blackwell; 2016.

19 Bassi C, Dervenis C, Butturini G, Fingerhut A, Yeo C, Izbicki J, Neoptolemos J, Sarr M, Traverso W, Buchler M and International Study Group on Pancreatic Fistula D: Postoperative pancreatic fistula: An International Study Group (ISGPF) definition. Surgery 138: 8-13, 2005.

20 Japanese Bureau of Medical Safety, Ministry of Health and Welfare: Guidelines for Administrating Blood Preparations. Medical and Pharmaceutical Notification No. 715, June 10, 1999.

21 Coussens LM and Werb Z: Inflammation and cancer. Nature 420: 860-867, 2002.

22 Grivennikov SI, Greten FR and Karin M: Immunity, inflammation and cancer. Cell 140: 883-899, 2010.

23 Karin M: Nuclear factor-kappaB in cancer development and progression. Nature 441: 431-436, 2006.

24 Heikkila K, Ebrahim S and Lawlor DA: A systematic review of the association between circulating concentrations of C-reactive protein and cancer. J Epidemiol Community Health 61: 824-833, 2007.

25 Mantovani A, Allavena P, Sica A and Balkwill F: Cancer-related inflammation. Nature 454: 436-444, 2008.

26 Ebrahimi B, Tucker SL, Li D, Abbruzzese JL and Kurzrock R: Cytokines in pancreatic carcinoma: Correlation with phenotypic characteristics and prognosis. Cancer 101: 2727-2736, 2004.
27 Forrest LM, McMillan DC, McArdle CS, Angerson WJ and Dunlop DJ: Evaluation of cumulative prognostic scores based on the systemic inflammatory response in patients with inoperable non-small-cell lung cancer. Br J Cancer 89: 10281030, 2003.

28 Crumley AB, McMillan DC, McKernan M, McDonald AC and Stuart RC: Evaluation of an inflammation-based prognostic score in patients with inoperable gastro-oesophageal cancer. $\mathrm{Br}$ J Cancer 94: 637-641, 2006.

29 Glen P, Jamieson NB, McMillan DC, Carter R, Imrie CW and McKay CJ: Evaluation of an inflammation-based prognostic score in patients with inoperable pancreatic cancer. Pancreatology 6: 450-453, 2006.

30 Leitch EF, Chakrabarti M, Crozier JE, McKee RF anderson JH, Horgan PG and McMillan DC: Comparison of the prognostic value of selected markers of the systemic inflammatory response in patients with colorectal cancer. Br J Cancer 97: 1266-1270, 2007.

31 Sohn TA, Yeo CJ, Cameron JL, Koniaris L, Kaushal S, Abrams RA, Sauter PK, Coleman J, Hruban RH and Lillemoe KD: Resected adenocarcinoma of the pancreas-616 patients: Results, outcomes and prognostic indicators. J Gastrointest Surg 4: 567$579,2000$.

32 Kusumanto YH, Dam WA, Hospers GA, Meijer C and Mulder $\mathrm{NH}$ : Platelets and granulocytes, in particular the neutrophils, form important compartments for circulating vascular endothelial growth factor. Angiogenesis 6: 283-287, 2003.

33 Barrientos S, Stojadinovic O, Golinko MS, Brem H and TomicCanic M: Growth factors and cytokines in wound healing. Wound Repair Regen 16: 585-601, 2008.

34 Smith RA, Bosonnet L, Raraty M, Sutton R, Neoptolemos JP, Campbell $\mathrm{F}$ and Ghaneh P: Preoperative platelet-lymphocyte ratio is an independent significant prognostic marker in resected pancreatic ductal adenocarcinoma. Am J Surg 197: 466-472, 2009.

Received October 2, 2018

Revised October 12, 2018

Accepted October 16, 2018 